\title{
Phenomenological Dynamics of Ising Magnets in the Presence of Magnetic Field: Widom Lines Captured from Slow Relaxation Time
}

\author{
S. ÖZÜM* \\ Alaca Avni Çelik Vocational School, Hitit University, 19600, Çorum, Turkey \\ Received: 14.06.2021 \& Accepted: 20.09.2021 \\ Doi: 10.12693/APhysPolA.140.252 \\ *e-mail: songulozum@hitit.edu.tr
}

\begin{abstract}
A complete phenomenological description for the relaxation dynamics of the Ising ferromagnets in the presence of external magnetic field $h$ is given by using the pair approximation. To study the relaxation dynamics, we calculate two characteristic times (denoted as $\tau_{S}, \tau_{F}$ ) using the rate constants which satisfy the Onsager reciprocity theorem. Temperature/magnetic field variations of $\tau_{S}$ and $\tau_{F}$ are investigated. We have shown that the extrema of the curves obtained for $\tau_{S}$ when $h \neq 0$ determine the loci of correlation length maxima or the Widom lines. The results are compared with those of response functions and thermodynamic curvature. We observe that they exactly match that for the magnetic susceptibility.
\end{abstract}

topics: relaxation times, Ising model, pair approximation, Widom line

\section{Introduction}

The Ising models have been the subject of important research for more than a century [1-20]. Among them, the phase transitions (PTs) driven by the spin-1/2 Ising magnets are one of the fundamental issues in equilibrium and nonequilibrium statistical mechanics. These transitions have been investigated theoretically by a variety of spin models such as the renormalization group method [21], MonteCarlo technique [22, 23], pair approximation [24-27] and effective field theory [28]. Although much attention has been devoted to the criticality and other thermodynamic properties, its time-dependent behaviors around the critical temperatures in such spin systems have been less studied. Especially, the study has focused on relaxation dynamics (RD).

The study of the RD begins with Barry who introduced phenomenologically the effects of flows in ordering fields on the simple kinetics and then extended the treatment into the steady-state kinetics [24]. Next, Meijer and co-workers [25] used the time-dependent pair approximation to study the relaxation of spin- $1 / 2$ Ising model in terms of the flow diagrams. Similarly, several investigations were concentrated on the RD and nonequilibrium critical behaviors in the same model using various techniques [29-35]. For the PTs theory and in particular the theory of $\mathrm{RD}$, the investigation of critical maxima gives an incredible effect [36]. A line, called the Widom line (WL), or loci of correlation length $(\xi)$ maxima, has been presented to describe the locus of maxima extending from the critical point [37]. The WL associated with the existence of a critical point has been especially mentioned in recent works $[38,39]$. An alternative definition for the WL is given in terms of the locus of maxima of the correlation length $\xi$. Emanating from any critical point there must be the loci of extrema of thermodynamic quantities, such as the Ricci curvature $(R)$, susceptibility $(\chi)$ and specific heat $\left(C_{H}\right)[27,40,41]$. Also, the relaxation time $\left(\tau_{S}\right)$ can be related to the correlation length $\xi[42,43]$ and so, related with the Widom line. The WL has been extensively studied which has recently provided information for theoretical studies of phase transitions and a method for the experimental data [44-48]. However, to our knowledge, there has been no study using the pair approximation focused on the relaxational dynamics related with the WL through the maxima of the correlation length $\xi$, that is captured by the relaxation time $\tau_{S}$.

In this study, we present a phenomenological description of the dynamics of a spin- $1 / 2$ Ising model with two ordering fields in the presence of external magnetic field. We use the ORT [49] together with the pair approximation (PA) for the free energy and investigate the temperature and magnetic field behaviors of slow and fast relaxation times near the continuous/discontinuous phase transition temperatures. The novelty of this paper is the broad extrema in the slow relaxation time near 
the critical point. Particularly, we have utilized the locus of a maximum of $\tau_{S}$ to find the Widom lines for the Ising model in the pair approximation in the $h-T$ plane.

\section{Description of model and equilibrium properties}

The spin- $1 / 2$ Ising model is defined by the Hamiltonian $[2,26,27]$

$$
H=-J \sum_{\langle i, j\rangle}^{N q / 2} \sigma_{i} \sigma_{j}-h \sum_{i}^{N} \sigma_{i} .
$$

Here, $\sigma_{i}= \pm 1 / 2$ in the $i$-th lattice site. The parameter $J$ is the exchange interaction for the nearestneighbor sites, and $N$ is the total number of lattice sites. The coordination number of the lattice is denoted by $q$ and $h=-g \mu_{B} H^{z}$ is the external magnetic field ( $H^{z}$ defined as the $z$-axis, $\mu_{B}$ as the Bohr magneton of each spin and $g$ - the electronic $g$ factor). The magnetic Gibbs energy per spin is written by the following form [26]

$$
\frac{G}{N}=-\frac{q}{2} J \psi-h \varphi-T\left[\frac{q}{2} S_{2}-(q-1) S_{1}\right],
$$

where $T$ is the absolute temperature, $\varphi=\left\langle\sigma_{i}\right\rangle$ is the single-site magnetization and $\psi=\varphi=\left\langle\sigma_{i} \sigma_{j}\right\rangle$ is the spin-pair correlation function for nearest-neighbor spins in (2). The single-site entropy $\left(S_{1}\right)$ and the two-site entropy $\left(S_{2}\right)$ are defined accordingly

$$
\begin{aligned}
S_{1} & =-k_{\mathrm{B}}\left[\left(\frac{1}{2}+\varphi\right) \ln \left(\frac{1}{2}+\varphi\right)\right. \\
& \left.+\left(\frac{1}{2}-\varphi\right) \ln \left(\frac{1}{2}-\varphi\right)\right],
\end{aligned}
$$

and

$$
\begin{aligned}
S_{2} & =-k_{B}\left[\left(\frac{1}{4}+\varphi+\psi\right) \ln \left(\frac{1}{4}+\varphi+\psi\right)\right. \\
& +2\left(\frac{1}{4}-\psi\right) \ln \left(\frac{1}{4}-\psi\right) \\
& \left.+\left(\frac{1}{4}-\varphi+\psi\right) \ln \left(\frac{1}{4}-\varphi+\psi\right)\right],
\end{aligned}
$$

where $k_{B}$ is Boltzmann's constant. Therefore, the conditions $\frac{\partial G}{\partial \varphi}=0, \frac{\partial G}{\partial \psi}=0$ yield the self-consistent equations

$$
\varphi=\frac{1}{2} \frac{\sinh (\alpha)}{\cosh (\alpha)+\mathrm{e}^{-\beta J / 2}},
$$

and

$$
\psi=\frac{1}{4} \frac{\cosh (\alpha)-\mathrm{e}^{-\beta J / 2}}{\cosh (\alpha)+\mathrm{e}^{-\beta J / 2}},
$$

at equilibrium with the notations

$$
\alpha=\frac{\beta}{q}[(q-1) r+h],
$$

where

$$
\begin{aligned}
& r=k_{\mathrm{B}} T \ln \left(\frac{\frac{1}{2}+\varphi}{\frac{1}{2}-\varphi}\right), \\
& \beta=\frac{1}{k_{\mathrm{B}} T} .
\end{aligned}
$$

The thermodynamical quantities, magnetic specific heat, isothermal susceptibility and the Ricci scalar have been studied by $\varphi$ and $\psi$ and discussed previously in [26, 27].

\section{Kinetic equations and relaxation times}

For the derivation of kinetic equations and relaxation times, the magnetic Gibbs energy is given in the neighborhood of equilibrium

$$
G(T, \varphi, \psi)=G^{(0)}\left(T, \varphi_{0}, \psi_{0}\right)+\Delta G,
$$

where $G^{(0)}\left(T, \varphi_{0}, \psi_{0}\right)$ is the equilibrium magnetic Gibbs energy in the absence of $h$. The terms of $\Delta G$ can be expressed in a Taylor series expansion of $G$ with respect to the spontaneous equilibrium point $\varphi=\varphi_{0}, \psi=\psi_{0}$. This is

$$
\begin{aligned}
& \Delta G=-\frac{1}{2}\left[\phi_{\varphi \varphi}\left(\varphi-\varphi_{0}\right)^{2}+\phi_{\psi \psi}\left(\psi-\psi_{0}\right)^{2}\right. \\
& \left.+2 \phi_{\varphi \psi}\left(\varphi-\varphi_{0}\right)\left(\psi-\psi_{0}\right)\right] .
\end{aligned}
$$

From (11) the expressions given below for $\phi_{\varphi \varphi}, \phi_{\varphi \psi}$, $\phi_{\psi \psi}$ are explicit functions of the $\varphi_{0}, \psi_{0}$ and take the form

$$
\begin{aligned}
\phi_{\varphi \varphi} & =\left(\frac{\partial^{2} G}{\partial \varphi^{2}}\right)_{\mathrm{eq}}, \\
\phi_{\varphi \psi} & =\left(\frac{\partial^{2} G}{\partial \varphi \partial \psi}\right)_{\mathrm{eq}}=\phi_{\psi \varphi}=\left(\frac{\partial^{2} G}{\partial \psi \partial \varphi}\right)_{\mathrm{eq}}, \\
\phi_{\psi \psi} & =\left(\frac{\partial^{2} G}{\partial \psi^{2}}\right)_{\mathrm{eq}} .
\end{aligned}
$$

Here, the subscript "eq" means equilibrium.

The generalized forces $X_{\varphi}, X_{\psi}$ may be written by using the Onsager reciprocity theorem (ORT) [49] with respect to $\varphi-\varphi_{0}, \psi-\psi_{0}$, respectively

$$
\begin{aligned}
& X_{\varphi}=\frac{\partial(\Delta G)}{\partial\left(\varphi-\varphi_{0}\right)}= \\
& -\phi_{\varphi \varphi}\left(\varphi-\varphi_{0}\right)-\phi_{\varphi \psi}\left(\psi-\psi_{0}\right), \\
& X_{\psi}=\frac{\partial(\Delta G)}{\partial\left(\psi-\psi_{0}\right)}= \\
& -\phi_{\psi \varphi}\left(\varphi-\varphi_{0}\right)-\phi_{\psi \psi}\left(\psi-\psi_{0}\right) .
\end{aligned}
$$

Also, the linear relations between the currents and forces can be given in terms of a matrix of phenomenological rate coefficients

$$
\left[\begin{array}{c}
\frac{\mathrm{d} \varphi}{\mathrm{d} t} \\
\frac{\mathrm{d} \psi}{\mathrm{d} t}
\end{array}\right]=\left[\begin{array}{cc}
\gamma_{\varphi} & -\gamma \\
\gamma & \gamma_{\psi}
\end{array}\right]\left[\begin{array}{c}
X_{\varphi} \\
X_{\psi}
\end{array}\right]
$$

By using (15) and (16) this matrix (17) yields the rate equations

$$
\begin{aligned}
\frac{\mathrm{d} \varphi}{\mathrm{d} t} & =-\Phi_{\varphi \varphi}\left(\varphi-\varphi_{0}\right)-\Phi_{\varphi \psi}\left(\psi-\psi_{0}\right), \\
\frac{\mathrm{d} \psi}{\mathrm{d} t} & =-\Phi_{\psi \varphi}\left(\varphi-\varphi_{0}\right)-\Phi_{\psi \psi}\left(\psi-\psi_{0}\right) .
\end{aligned}
$$

The coefficients are defined by

$$
\Phi_{\varphi \varphi}=\gamma_{\varphi}\left(\frac{\partial^{2} G}{\partial \varphi^{2}}\right)_{\mathrm{eq}}-\gamma\left(\frac{\partial^{2} G}{\partial \varphi \partial \psi}\right)_{\mathrm{eq}},
$$




$$
\begin{aligned}
& \Phi_{\varphi \psi}=\gamma_{\varphi}\left(\frac{\partial^{2} G}{\partial \varphi \partial \psi}\right)_{\mathrm{eq}}-\gamma\left(\frac{\partial^{2} G}{\partial \psi^{2}}\right)_{\mathrm{eq}}, \\
& \Phi_{\psi \varphi}=\gamma\left(\frac{\partial^{2} G}{\partial \varphi^{2}}\right)_{\mathrm{eq}}+\gamma_{\psi}\left(\frac{\partial^{2} G}{\partial \varphi \partial \psi}\right)_{\mathrm{eq}}, \\
& \Phi_{\psi \psi}=\gamma_{\psi}\left(\frac{\partial^{2} G}{\partial \psi^{2}}\right)_{\mathrm{eq}}+\gamma\left(\frac{\partial^{2} G}{\partial \varphi \partial \psi}\right)_{\mathrm{eq}} .
\end{aligned}
$$

A solution of $\mathrm{e}^{-t / \tau}$ for (18) and (19), the secular equation, is written as

$$
\left|\begin{array}{cc}
\tau^{-1}-\Phi_{\varphi \varphi} & -\Phi_{\varphi \psi} \\
-\Phi_{\psi \varphi} & \tau^{-1}-\Phi_{\psi \psi}
\end{array}\right|=0
$$

which gives two relaxation times $[50,51]$

$$
\begin{aligned}
\frac{1}{\tau_{S}} & =\frac{\Phi_{\varphi \varphi} \Phi_{\psi \psi}-\Phi_{\varphi \psi} \Phi_{\psi \varphi}}{\Phi_{\varphi \varphi}+\Phi_{\psi \psi}}, \\
\frac{1}{\tau_{F}} & =\Phi_{\varphi \varphi}+\Phi_{\psi \psi}-\frac{1}{\tau_{S}} .
\end{aligned}
$$

Here, $\tau_{S}$ is the slower and $\tau_{F}$ is the faster relaxation time. They are related to the single-site magnetization and the spin-pair correlation parameter, respectively.

\section{Results and discussion}

In this part, our relaxational quantities $\left(\tau_{S}, \tau_{F}\right)$ are plotted for the Ising model with the PA in the ferromagnetic (FM) range, the paramagnetic (PM) range and exactly at the continuous phase transition temperature. The thermal and magnetic field behaviors for $\tau_{S}$ and $\tau_{F}$ are presented, respectively, in Figs. 1-3. The phenomenological rate constants $\gamma_{\varphi}=1, \gamma=0.00001, \gamma_{\psi}=10$ are used in these figures. Particularly, we have examined the loci of maxima, the WL, of dynamic and thermodynamic quantities.

Firstly, schematic illustrations of the thermal behaviors for $\tau_{S}$ using various field values $h$ and lattice coordination numbers $q$ and the corresponding WL related to $\tau_{S}$ are presented in Fig. 1. A comparison of the WL related with some of thermodynamic quantities, such as the Ricci curvature $R$, the static magnetic susceptibility $\chi$ and the specific heat $C_{H}$, are also included in Fig. 1c using the simple cubic lattice with $q=6$ and $J=1$. The slow relaxation time $\tau_{S}$ versus temperature calculations that have been obtained for four values of the external fields $h=0,0.03,0.05,0.1$ for $J=1$ and $q=6$ and for various values of $q=4,6,8$ with $h=0.05$, $J=1$ are displayed in Fig. 1a and b, respectively. In Fig. 1a, $\tau_{S}$ grows rapidly with increasing temperature and diverges to infinity around critical temperature $T_{\mathrm{c}}$ as can be seen from the green-colored curve when $h=0$. This behavior is similar to the relaxation phenomena in Barry's works and Tanaka and Takahashi's works [24, 52]. The divergence of $\tau_{S}$ has not been seen and maxima of the curves (or peaks) are obtained when $h \neq 0$. In particular, for the cases of $\tau_{S}$, these maxima depend on the external field. One can see that with the increase
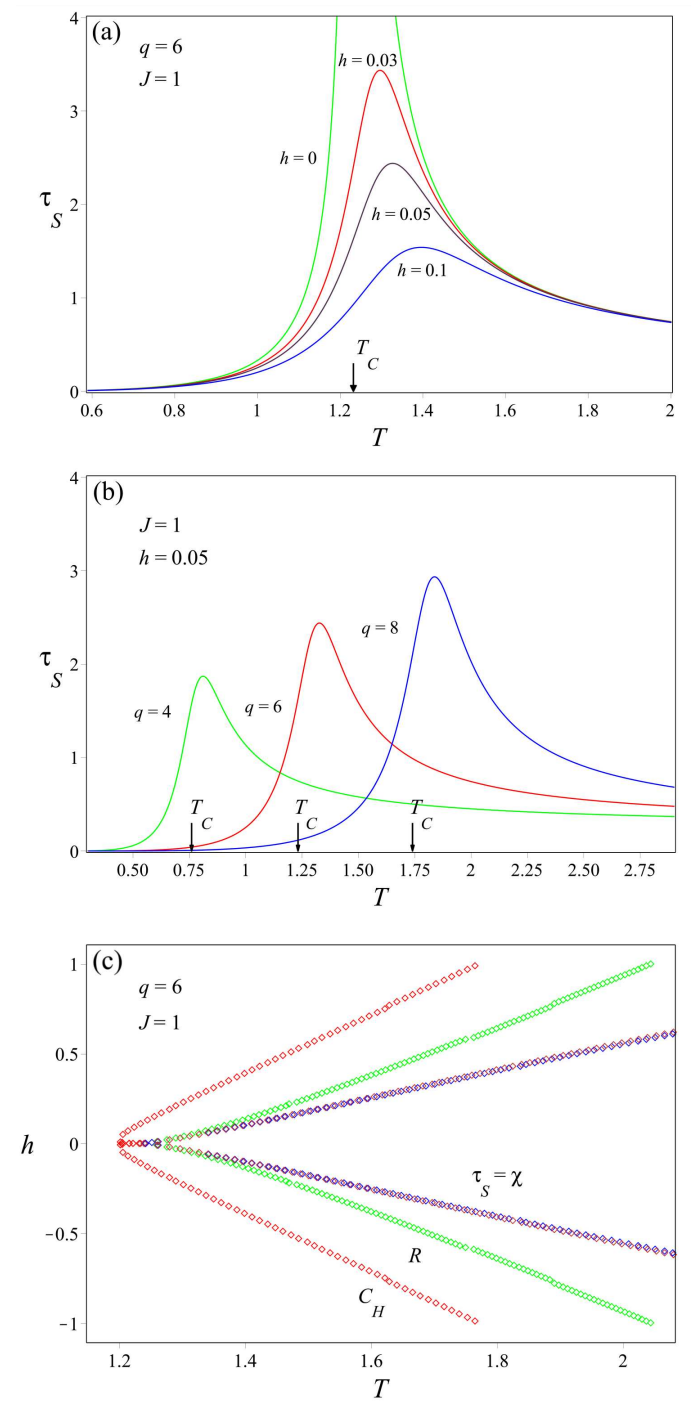

Fig. 1. (a) The $\tau_{S}-T$ curves calculated for various values of $h$ with $q=6$ and $J=1$. (b) Description used is the same as in (a) but for various values of $q$ with $h=0.05$ and $J=1$. (c) Loci of maxima of $C_{H}$ (red-colored circle), $R$ (green-colored circle), $\chi$ (blue-colored circle) and $\tau_{S}$ (orange-colored circle) for $q=6$ and $J=1$ on the $h-T$ plane.

of $h(h=0.03,0.05$, and 0.1$)$ the maxima become smaller and shift towards higher temperatures. It is important to mention that a coherent behavior of $\tau_{S}$ versus $T$ in Fig. 1a is also observed in the $\chi-T$ plots in [26] and the relaxation times in [13, 53, 54].

For comparison, we have also displayed $\tau_{S}$ versus $T$ for this system with $q=4$ (square lattice), $q=6$ (simple cubic lattice) and $q=8$ (body centered cubic lattice) in Fig. 1b. We have used $h=0.05$ in this figure. Upon increasing $q$ values, the peaks become larger and shift towards higher temperatures. Furthermore, in Fig. 1c, the extrema of the curves investigated for $h \neq 0$ determine the loci of the correlation length maxima or the Widom lines obtained from Fig. 1a, predicted for the Ising model 
in PA on the $h-T$ plane. For comparison, we observed two Widom lines for $\tau_{S}, \chi, R$, and $C_{H}$ that start at the critical points and extend into the regions $h>0$ and $h<0$ in the $h-T$ plane in Fig. 1c. We compare our results and find important agreement for the Widom lines with the mean-field CW model [27, 44].

We have also presented $\tau_{F}$ versus $T$ for the same system in Fig. 2a and b. The present model predicts that both the position and shape of the curves are different when the magnetic field $h$ is changed (the same $h$ values in Fig. 1a). For example, when the $h$ value decreases, the $\tau_{F}$ versus $T$ curve is shifted to lower temperature and the slope at $T_{\mathrm{c}}$ becomes steeper, as seen in Fig. 2a. When the $h$ value increases, this curve is shifted to higher temperature and the slope becomes less steep. In other words, the curves show a field-dependent behavior when $h \neq 0$ in Fig. 2a. Figure 2b shows the $\tau_{F}$ versus $T$ for the same system with $q=4,6$, and 8 . In this figure, $h=0.05$ is used for all lattices. The value of $\tau_{F}$ is decreasing with increasing lattice coordination number $q$ and shift towards higher temperatures.

To demonstrate $\tau_{S}$ and $\tau_{F}$ as a function of $h$, we change the $T$ values in Fig. 3 with $q=6$ and $J=1$. In this figure, $T=1.180\left(T<T_{\mathrm{c}}\right.$, blue-colored curve), $T=T_{\mathrm{c}} \approx 1.232$ (green-colored curve) and $T=1.280\left(T>T_{\mathrm{c}}\right.$, red-colored curve) are given. The $\tau_{S}$ and $\tau_{F}$ are positive and symmetric on both
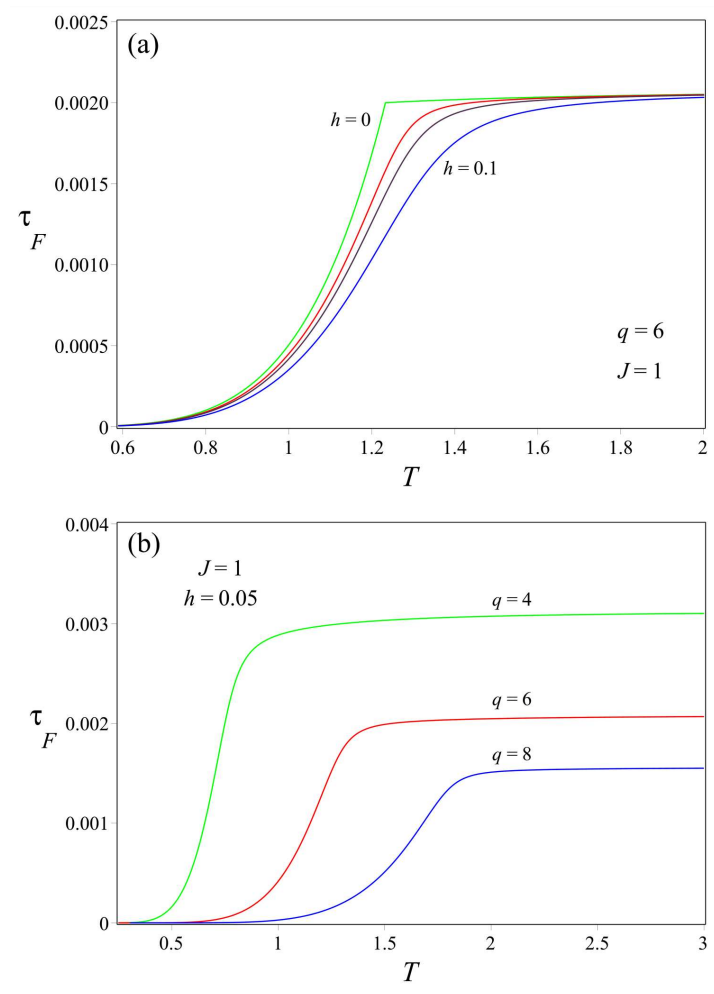

Fig. 2. (a) The $\tau_{F}-T$ curves calculated for various values of $h$ with $q=6$ and $J=1$. (b) Description used is the same as in (a), but for various values of $q$ with $h=0.05$ and $J=1$.
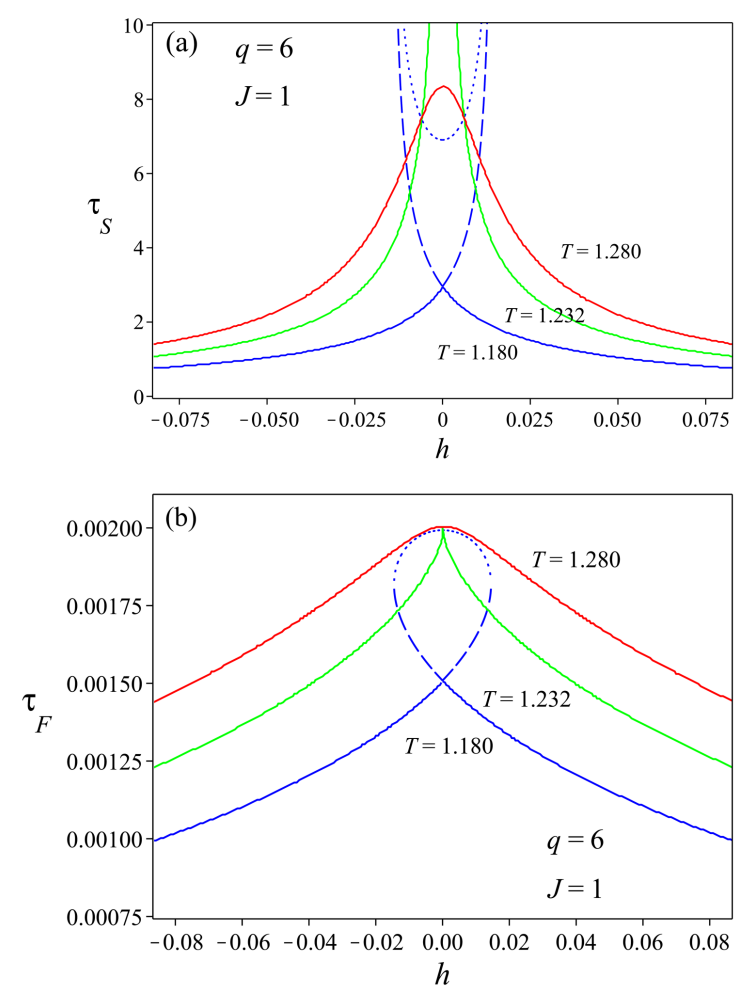

Fig. 3. The $\tau_{S}-h$ and $\tau_{F}-h$ curves for various values of $T$ with $q=6$ and $J=1$.

sides of the $h=0$ line for all $T$ values which is also similar to the behavior of the curvature function by Erdem [27]. These results mean that relaxation times and the curvature scalar are independent of orientation of the $h, \tau_{S}(-h)=\tau_{S}(h)$ and $\tau_{F}(-h)=\tau_{F}(h)$. In the FM phase, $T<T_{\mathrm{c}}$, the $\tau_{S}$ and $\tau_{F}$ behave differently (has two physical branches) which cross at $h=0$. Again, the $\tau$ crossing occurred below the criticality. In turn, in $T=T_{\mathrm{c}}$, a divergence to infinity in $\tau_{S}$ appears again, as also seen in Fig. 1a. In the PM phase, $T>T_{\mathrm{c}}$, there is no crossing, but the $\tau_{S}$ shows a maximum at $h=0$ in Fig. 3 .

\section{Conclusion}

In this study, we have shown that the relaxational dynamics are related to the Widom line of the Ising model. Firstly, we obtained and showed the existence of two different time scales as slow $\tau_{S}$ and fast $\tau_{F}$ relaxation times with using the approach based on two order parameters $(\varphi, \psi)$. In fact, relaxation time $\tau_{S}$ may be connected to the correlation length, $\xi \approx\left|T-T_{\mathrm{c}}\right|^{-v}$, by $\tau_{S} \approx \xi^{v}$, where $v$ is the correlation length exponent, $T$ is the temperature and $T_{\mathrm{c}}$ is the critical value. The relaxation times $\tau_{S}$ and $\tau_{F}$ behave differently (have two physical branches) that shows the $\tau$-crossing at $h=0$ near the phase transitions in the $\tau_{S}-h / \tau_{F}-h$ planes. The dependencies $\tau_{S}$ vs $T$ and $\tau_{S}$ vs $h$ have singularities (divergence) at the critical point. The findings for relaxation times versus temperature are in 
accordance with the other results obtained as theoretical [52, 54-57] and experimental [58-60]. Based on the previous discussions [44], we have investigated the lines of the maxima of $\tau_{S}, \chi, R$ and $C_{H}$ in the phase region, the so-called WL. In this paper, we have tried to show the connection between the WL and the thermodynamical/dynamical behavior. Finally, it would be of great interest in future work as disciplines in physical, chemical, and biological sciences and engineering [61].

\section{References}

[1] W. Lenz, Z. Phys. 21, 613 (1920).

[2] E. Ising, Z. Phys. 31, 253 (1925).

[3] L. Onsager, Phys. Rev. 65, 117 (1944).

[4] C.N. Yang, Phys. Rev. 85, 808 (1952).

[5] P. Pfeuty, Ann. Phys. 57, 79 (1970).

[6] H.C. Fogedby, J. Phys. C 11, 2801 (1978).

[7] C.A. Basha, M.V. Sangaranarayanan, J. Electroanal. Chem. Interfac. Electrochem. 291, 261 (1990).

[8] A. Ainane, M. Saber, F. Dujardin, B. Stébé, Surf. Sci. 482-485, 1068 (2001).

[9] J. Strečka, Phys. Lett. A 349, 505 (2006).

[10] J. Strečka, O. Rojas, S.M. de Souza, Phys. Lett. A 376, 197 (2012).

[11] C.K. Hu, Chin. J. Phys. 52, 1 (2014).

[12] M.R. Soltani, J. Vahedi, S. Mahdavifar, Physica A 416, 321 (2014).

[13] R. Erdem, G. Gulpınar, O. Yalçın, A. Pawlak, J. Appl. Phys. 116, 034908 (2014).

[14] I.J.L. Diaz, N.S. Branco, Physica B 529, 73 (2018)

[15] J. Kplé, S. Massou, F. Hontinfinde, E. Albayrak, Chin. J. Phys. 56, 1252 (2018).

[16] H.K. Mohamad, Chin. J. Phys. 56, 1547 (2018).

[17] F. Souza, M.L. Lyra, J. Strečka, M.S.S. Pereira, J. Magn. Magn. Mater. 471, 423 (2019).

[18] I.J.L. Diaz, N.S. Branco, Physica A 540, 123014 (2020).

[19] L. Gálisová, Phys. Lett. A 384, 126629 (2020).

[20] L. Gálisová, J. Strečka, T. Verkholyak, S. Havadej, Physica E 125, 114089 (2021).

[21] A.N. Berker, Phys. Rev. B 12, 2752 (1975).

[22] O.G. Mouritsen, J. Phys. C 13, 3909 (1980).

[23] J.-S. Wang, C.K. Gan, Phys. Rev. E 57, 6548 (1998).

[24] J.H. Barry, J. Chem. Phys. 45, 4172 (1966).
[25] P.H.E. Meijer, M. Keskin, E. Bodegom, J. Stat. Phys. 45, 215 (1986).

[26] T. Balcerzak, Physica A 317, 213 (2003).

[27] R. Erdem, Physica A 526, 121173 (2019).

[28] A. Oubelkacem, A. Ainanea, J.J. de Miguel, J.R. de Sousa, M. Saber, Physica A 358, 160 (2005).

[29] J.J. Brey, A. Prados, Europhys. Lett. 57, 171 (2002).

[30] G. Gulpinar, D. Demirhan, F. Buyukkilic, Phys. Rev. E 75, 021104 (2007).

[31] G. Gulpinar, Y. Karaaslan, Phys. Lett. A 375, 978 (2011).

[32] G. Gulpinar, E. Vatansever, J. Magn. Magn. Mater. 324, 983 (2012).

[33] M. Ertaş, M. Keskin, Phase Transit. 87, 376 (2014).

[34] S. Goh, J.-Y. Fortin, M.Y. Choi, Physica A 466, 166 (2017).

[35] W.L. de Souza, É. de Mello Silva, P.H.L. Martins, Phys. Rev. E 101, 042104 (2020).

[36] G. Camisasca, M. De Marzio, M. Rovere, P. Gallo, Entropy 19, 185 (2017).

[37] P. Gallo, D. Corradini, M. Rovere, Nat. Commun. 5, 1 (2014).

[38] V.V. Brazhkin, Y.D. Fomin, A.G. Lyapin, V.N. Ryzhov, E.N. Tsiok, J. Phys. Chem. $B$ 115, 14112 (2011).

[39] S. Han, C.C. Yu, Phys. Rev. E 85, 051201 (2012).

[40] L. Xu, P. Kumar, S.V. Buldyrev, S.-H. Chen, P.H. Poole, F. Sciortino, H.E. Stanley, Proc. Natl. Acad. Sci. 102, 16558 (2005).

[41] P. Kumar, Z. Yan, L. Xu, M.G. Mazza, S.V. Buldyrev, S.-H. Chen, S. Sastry, H.E. Stanley, Phys. Rev. Lett. 97, 177802 (2006).

[42] M. Ouarch, M. Benhamoua, M. Chahid, H. Kaidi, Eur. Phys. J. E 29, 319 (2009).

[43] G. Gulpinar, F. Iyikanat, Phys. Rev. E 83, 041101 (2011).

[44] A. Dey, P. Roy, T. Sarkar, Physica A 392 , 6341 (2013).

[45] B. Widom, in: Phase Transitions and Critical Phenomena, Vol. 2, Eds. C. Domb, M.S. Green, Academic Press, 1972.

[46] K.A. Dawson, Phys. Rev. A 36, 3383 (1987).

[47] K.A. Dawson, M.D. Lipkin, B. Widom, J. Chem. Phys. 88, 5149 (1988).

[48] G.G. Simeoni, T. Bryk, F.A. Gorelli, M. Krisch, G. Ruocco, M. Santoro, T. Scopigno, Nat. Phys. 6, 503 (2010). 
[49] L. Onsager, Phys. Rev. 37, 405 (1931); 38, 2265 (1931).

[50] R. Erdem, S. Özüm, Mod. Phys. Lett. B 33, 1950258 (2019).

[51] S. Özüm, R. Erdem, Mod. Phys. Lett. B 34, 2050338 (2020).

[52] M. Tanaka, K. Takahashi, Prog. Theor. Phys. 58, 387 (1977).

[53] M. Keskin, R. Erdem, Phys. Lett. A 297. 427 (2002).

[54] R. Erdem, C. Ekiz, M. Keskin, Phys. Status Solidi B 240, 220 (2003).

[55] P. Goswami, Phys. Rev. B 33, 6094 (1986).
[56] V.V. Prudnikov, A.V. Ivanov, A.A. Fedorenko, J. Exp. Theor. Phys. Lett. 66, 835 (1997).

[57] D. Ivaneyko, J. Ilnytskyi, B. Berche, Y. Holovatch, Physica A 370, 163 (2006).

[58] D.P. Belanger, B. Farago, V. Jaccarino, A.R. King, C. Lartigue, F. Mezei, J. Phys. (Paris) Colloq. 49, C8 (1988).

[59] N. Rosov, C. Hohenemser, M. Eibschutz, Phys. Rev. B 46, 3452 (1992).

[60] P. Grassberger, Physica A 214, 547 (1995); Erratum: 217, 227 (1995).

[61] G. Ruppeiner, A. Sahay, T. Sarkar, G. Sengupta, Phys. Rev. E 86, 052103 (2012). 